\title{
Arrhythmogenic Convulsive Syncope in Neurological Practice: A Case Report
}

\author{
Diana V. Dmitrenko, $\mathrm{PhD}, \mathrm{ScD}^{1 *}$; Irina G. Strotskaya, $\mathrm{MD}^{1}$; Ivan P. Artyukhov, $\mathrm{PhD}, \mathrm{ScD}^{1}$; \\ Olga V. Alekseeva, MD; Natalia A. Shnayder, PhD, ScD ${ }^{1}$; Gennady V. Matyushin, PhD, $\mathrm{ScD}^{1}$; \\ Vladimir V. Shprakh, PhD, ScD²; Svetlana B. Sayutina, PhD²; Oksana A. Gavrilyuk, $\mathrm{PhD}^{1}$ \\ ${ }^{1}$ Krasnoyarsk State Medical University named after Prof. V.F.Voino-Yasenetsky, Krasnoyarsk \\ ${ }^{2}$ Irkutsk State Medical Academy of Postgraduate Education, Irkutsk \\ the Russian Federation
}

\begin{abstract}
This case report presents a 24-year-old man with a long history of arrhythmogenic convulsive syncope, which was managed as a cryptogenic generalized pharmacoresistant epilepsy (PRE). During complex examination of the patient within the framework of preoperative screening for the purpose of neurosurgical treatment of PRE, an idiopathic SSS was diagnosed. The clinical diagnosis was changed. The patient underwent emergency surgery at the center for cardiac surgery. ECP was implanted, the seizures stopped, resulting in dramatically improved quality of life.(International Journal of Biomedicine. 2018;8(1):65-68.)
\end{abstract}

Key words: epilepsy $\bullet$ seizure, syncope $\bullet$ sick sinus syndrome $\bullet$ cardiac rhythm disorders $\bullet$ management

\section{Abbreviations}

ECG, electrocardiography; ECP, electric cardiac pacemaker; EEG, electro-encephalography; HR, heart rate; MRI, magnetic resonance imaging; PRE, pharmacoresistant epilepsy; SSS, sick sinus syndrome.

\section{Introduction}

Differential diagnosis of arrhythmogenic convulsive syncope and generalized tonic-clonic seizures represents a complicated interdisciplinary problem of clinical medicine, ${ }^{(1)}$ since convulsive syncope is often diagnosed as epileptic seizures: In cases of temporary loss of consciousness with convulsions, a major epilepsy diagnosis does not admit of doubt among the majority of primary care physicians. ${ }^{(2)}$ All this leads to a long-term and ineffective prescription of antiepileptic drugs, misdiagnosis of PRE, and patients' referral to neurosurgical treatment. ${ }^{(3)}$

A syncopal condition (syncopal attack, syncope) represents a temporary loss of consciousness due to general

*Corresponding author: Diana V. Dmitrenko, PhD, ScD Neurological Center for Epileptology, Neurogenetics and Brain Research. Krasnoyarsk State Medical University n.a. Prof. V.F. Voino-Yasenetsky, Krasnoyarsk the Russian Federation.E-mail: mart2802@yandex.ru cerebral hypoperfusion, characterized by a rapid development, short duration and spontaneous remission. ${ }^{(4)}$ The problems of managing major convulsive syncope include refusal of or technical impossibility of conducting long-term video-electroencephalographic monitoring with parallel implementation of ECG monitoring, lack of an interdisciplinary approach to following up and differential diagnostics, and refusal to conduct additional patient-specific, load-adaptive testing that models a trigger situation for the development of an arrhythmogenic convulsive syncope. Our clinical observation of a young male patient who was followed up, together with that of our colleagues is indicative of the abovementioned.

\section{Case Presentation}

In June 2016, a 26-year-old male diagnosed with cryptogenic generalized PRE was admitted to the Neurological Center of Epileptology, Neurogenetics and Brain Research of the 
University Clinic of Prof. V.F. Voino-Yasenetsky Krasnoyarsk State Medical University for a pre-surgical examination and adjudication in the matter of further neurosurgical treatment in the Federal Center.

At the initial consultation, the patient presented problems with seizures during the medical manipulations or discussion of medical procedures and events. The seizures started from dizziness, shortness of breath and fluctuations in the level of consciousness for periods up to 2 minutes with subsequent loss of consciousness, skin pallor and single-time clonic convulsions in the upper limbs with periodic urinary incontinence. The convulsion duration did not exceed one minute and no tongue biting was present. There had been no control over either blood pressure level or HR and blood sugar level during seizures. The patient had received long-lasting antiepileptic treatment with lamotrigine (50 $\mathrm{mg}$ per day) for one year previously with no clinical effect.

The patient's medical history showed that perinatal anamnesis was not burdened; early psychomotor and speech development conformed to the patient's age. At the age of 3 to 5 months, affective respiratory attacks were registered. At the age of 3, an episode with loss of consciousness and a fall without convulsions was registered for the first time while the patient was viewing an abdominal surgery on television. Syncope was mainly registered during undertaking of medical procedures and/or discussion of the revealed results as well as during discussions about any medical topics and issues, including complaints of the patients' relatives about the state of his health. Seizures with loss of consciousness and convulsions were registered at the ages of $9,12,13-14,17-18,23$, and 25 years. In 2016, 4 events were registered, including seizures with involuntary urination, and generalised myoclonic seizures in the upper and lower limbs, which occurred when the patient saw blood or was present during discussions about results of examinations of his health or health of his relatives. The patient denied having a hereditary history of epilepsy or cardiac rhythm disorders. Electrophysiological examinations of first- and second-degree relatives was not conducted. A cranial-cerebral injury at the age of 6 was present in his medical history.
Objective data: the patient had a satisfactory state, clear consciousness, and preserved intellectual function; he was oriented, critical and emotionally labile. Levels of the state and trait anxiety were elevated. The patient was of normosthenic constitution. Skin cover was smooth, normal color and with no peripheral oedema. No acute distress was observed in his somatic status. In the neurological status, during examination, no symptoms of brain lesions were revealed.

According to data obtained during repeated routine EEG, epileptiform activity was not registered; the ECG channel was turned off during the EEG procedure. The level of lamotrigine in the blood was subtherapeutic $(2.44 \mathrm{ug} / \mathrm{ml})$. In order to specify the diagnosis, the patient underwent complete electrophysiological, neurophysiological and neuroradiological examination. An MRI of the brain (1.5 Tesla) according to the epilepsy protocol revealed a single locus of leukoaraiosis $(0.2 \mathrm{~cm})$ on the left frontalsubcortical parts of the insula, mild asymmetry of hippocampi $\mathrm{D}>\mathrm{S}(10 \%)$ without visible structural alterations.

Considering the provoked character of his seizures, the patient was given long-term video-EEG monitoring with parallel Holter-ECG registration with amplified exercise testing, including partial sleep deprivation with a forced wake-up test. Ictal and interictal epileptiform activity were not registered in passive wakefulness or during sleeping and standard physical exercise tests. In this connection, low-trauma invasive manipulation was performed (the patient lying on his back, venous blood sampling from the cubital vein) for the purpose of modelling the clinical situation that was a trigger to a convulsive seizure. During this time, tachycardia with HR of up to $120 \mathrm{bpm}$ for 10 seconds was registered with subsequent lowering of HR to $45 \mathrm{bpm}$ during 15 seconds and asystole for 15 seconds. During parallel video-EEG monitoring, the patient had a seizure of consciousness impairment with mainly leftsided myoclonic seizures in the lower limbs lasting for up to 2 seconds. During the seizure, verbal contact with the patient was unavailable. Upon analysis of the channel in II standard lead of the ECG before loss of consciousness, an RR pause of $15540 \mathrm{~ms}$ was registered with subsequent recovery of the sinoatrial rate (Fig.1).

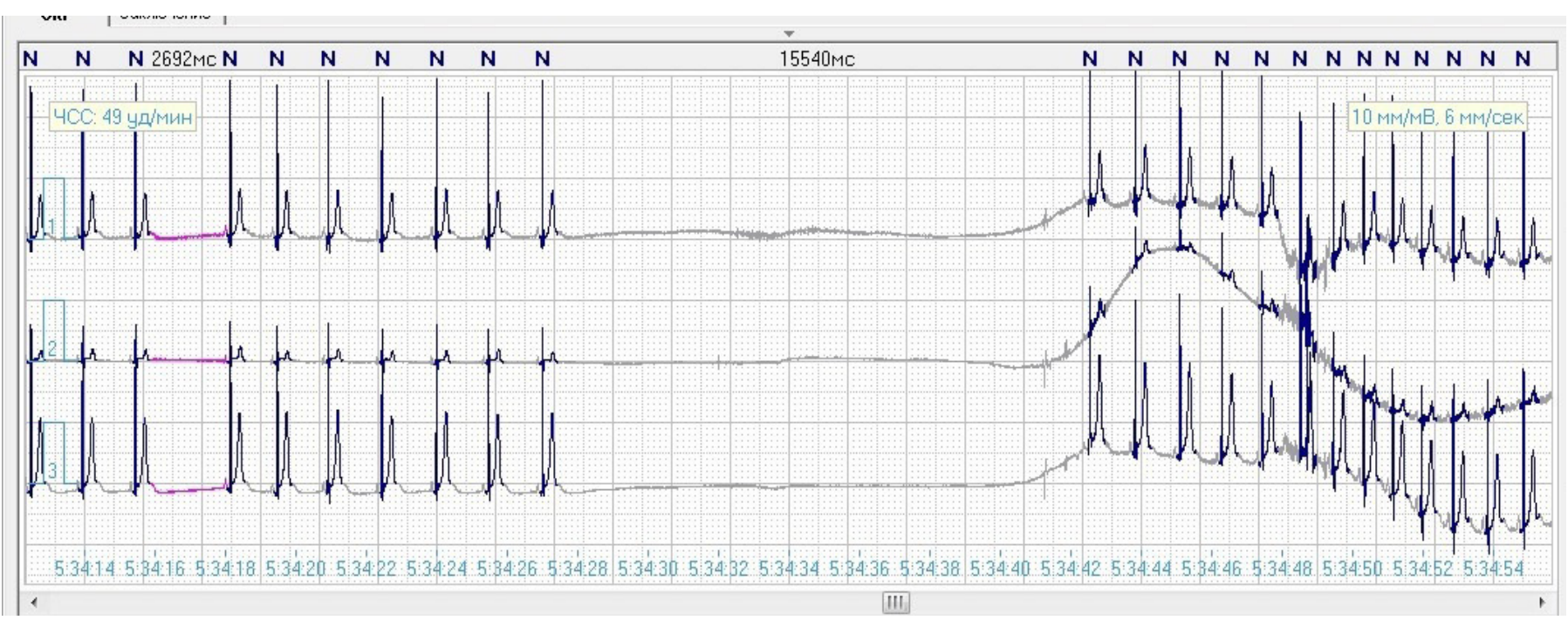

Fig. 1. Fragment of the ECG with RR pause of $15540 \mathrm{~ms}$. 
According to EEG data, diffuse delta waves (as a marker of a decrease in cortical neuron functional activity) were registered for 4 seconds during consciousness impairment and sinus arrest. Thereafter, defuse polyspike-wave activity with an amplitude of $70 \mathrm{uV}$ was registered for one second (the pattern of a myoclonic seizure), as well as motor artifacts with a duration of 5 seconds with subsequent polymorphous low-amplitude bioelectric activity (Fig.2). Total duration of the paroxysm was 30 seconds. Blood pressure after the paroxysm was $135 / 70 \mathrm{mmHg}$.

Taking data of the pre-surgical examination into account, clinical diagnosis was re-established as cardiogenic (arrhythmogenic) generalized myoclonic seizures. SSS? Sinoatrial node arrest.

The patient was consulted by a cardiologist-arrhythmologist during the first 24 hours after development of the abovedescribed seizure. A diagnosis was established for the first time: Idiopathic SSS and transient sinus arrest. The patient underwent emergency hospitalization in the Krasnoyarsk Federal Centre of Cardiovascular Surgery where he was implanted with a bicameral ECP. The patient's state was satisfactory; antiepileptic therapy was cancelled against this background.
According to data obtained from 3 hours of video-EEGmonitoring after sleep deprivation for 24 hours with parallel HolterECG monitoring after 3 months following ECP implantation, ictal and interictal epileptiform activity was not registered in the state of passive wakefulness, during exercise tests, and in the slow wave sleep phase. During an exercise test with intravenous injection imitation, HR increased up to $90 \mathrm{bpm}$ (while sitting). Lowamplitude activity in the alpha-two frequency range was registered by means of EEG monitoring; beta-activity showed no alteration in the consciousness level or behaviour of the patient. However, a short episode of consciousness impairment with slackening and without seizures, tongue biting or involuntary urination, and with registration of second-degree AV block with RR pause up to 2004 $\mathrm{ms}$ (ECP detection function compromise was not improbable) was registered in the patient during Holter-ECG monitoring (after 24 hours of sleep deprivation).

Catamnesis after 12 months: the patient's state is satisfactory, no repeated bouts, life quality and emotional state of the patient have improved significantly. The patient has received repeated consultations by a cardiologist-arrhythmologist; no seizures were registered within the past 12 months.

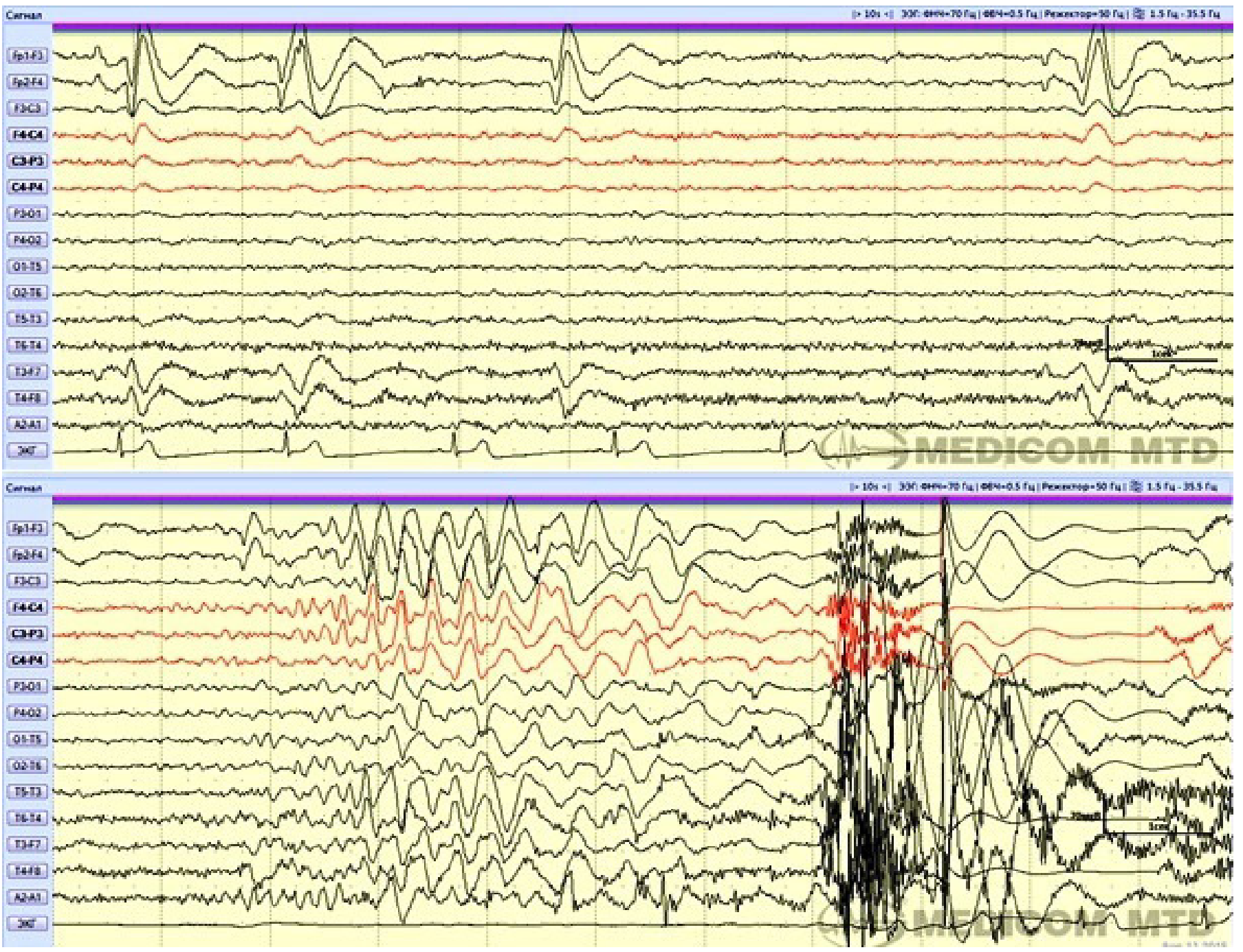

Fig. 2. Fragment of video EEG monitoring: lowering of HR to $45 \mathrm{bpm}$ during 15 seconds and asystole for 15 seconds. Fragment of EEG: high-amplitude polyspike-wave activity with an amplitude of $70 \mathrm{uV}$ for one second, as well as motor artifacts with a duration of 5 seconds with subsequent polymorphous low-amplitude bioelectric activity. 


\section{Discussion}

Differential diagnosis of seizures leading to loss of consciousness is very extensive. In general, an examination is carried out to exclude epilepsy, metabolic disorders, transient ischemic attacks in the posterior circulation system (or drop attacks), narcolepsy and psychogenic pseudosyncopes. ${ }^{(2)}$ Usually, differential diagnosis is not difficult in such cases, although sometimes it can be complicated by insufficient anamnesis, atypical symptoms or actual complexity in syncope identification. ${ }^{(5)}$

Clinical manifestations of the generalized epileptic seizure can be similar to arrhythmogenic convulsive syncope and include fainting/precollaptoid state, visual and audial disorders, convulsions, tongue biting and involuntary urination. Other possible syncope symptoms comprise epigastric aura with abdominal discomfort and/or unusual foul smells, diffuse hyperhidrosis and skin pallor. ${ }^{(6)}$ Involuntary motions in limbs, urination and tongue biting might be present in both epilepsy and syncope. Modern video-analysis (video-EEG monitoring) makes it possible to state that $90 \%$ of patients have myoclonic convulsions, oral automatism symptoms and oculogyric deviation during syncope. Therefore, such patients have clinical manifestations which can be misdiagnosed as epilepsy.(7) Generally, post-seizure disorientation in time and space is absent after a syncope episode. However, retrograde amnesia might occur more often in such cases than had been suggested before, especially in aged patients. Sometimes the postsyncopal period includes manifestations of general weakness and somnolence. ${ }^{(8)}$

Cardiac rhythm disorders are the most frequent reasons for convulsive syncope as they induce hemodynamic impairments leading to critical lowering of cardiac output and cerebral blood flow. Nevertheless, syncope may be caused by such multiple accessory factors as HR, arrhythmia type (supraventricular or ventricular), myocardial function of the left ventricle, body position and adequacy of vascular compensatory reactions. The latter include baro-receptor nervous reflexes as well as reflex response to eye opening induced by arrhythmia. In cases of SSS, there is damage to the sinoatrial node due to impairment of its automatism or sinoatrial conduction. In this situation, syncope is conditioned by long pauses resulting from sinus node arrest or sinoatrial block and insufficiency of second-order replacing centers. Similar pauses are most often developed after a sudden stop of atrial tachyarrhythmia paroxysm (tachy-brady syndrome). ${ }^{(4)}$

Arrhythmogenic syncope is an independent factor in risk of sudden death syndrome. ${ }^{(9)}$ The difficulty of diagnosing idiopathic heart rhythm disorders is conditioned by their oligosymptomatic or asymptomatic progression and frequent total absence of representative data from a physical examination. Among high-risk factors, the presence of which requires immediate hospitalisation or intensive treatment, there are clinical and ECG syncope symptoms of arrhythmogenic aetiology, including a syncope seizure during tension or in a supine position, and an increase in HR during syncope. ${ }^{(4)}$

Most critical elements in the differential diagnosis of epilepsy and convulsive syncope are medical case history, triggers, presence and/or absence of other types of epileptic seizures, and epileptiform activity in EEG scans. At the same time, the absence of epileptiform activity in EEG scans, especially against the background of a single-step examination, still does not exclude the possibility of the seizure having an epileptic nature. ${ }^{(6)}$ Differential diagnostics requires participation of a cardiologist-arrhythmologist in the patient's examination. A tilt-test (or tilt-table test), Holter-ECG monitoring, and eventmonitor implantation, as well as distant-recording thermometry, can be used on therapeutic grounds..$^{(3,4,10)}$

\section{Conclusion}

Thus, differential diagnostics of convulsive syncope and epileptic seizures deserves the focused attention of clinicians and requires an interdisciplinary approach to ensure early diagnosis and appropriate treatment, improve the quality of life and reduce the risk of life-threatening conditions, particularly sudden death syndrome.

\section{Competing interests}

The authors declare that they have no competing interests.

\section{References}

1. Tereshchenko SY, Evert LS, Lytkin VA, Bobrova EI. [Cardiogenic syncopal conditions in children and teenagers: the problems of differential diagnosis]. Siberian Medical Review. 2010;65(5):88-94. [Article in Russian].

2. Usacheva EL, Osipova KV, Prityko AG. [Clinical features of non-epileptic seizures in children]. Nevrologicheskii Zhurnal. 2009;1:18-24. [Article in Russian].

3. Mironov MB. [Analysis of main reasons for mistakes in diagnostics of epileptic seizures and epileptic syndromes (clinical peculiarities of epileptic seizures)]. Russian Journal of Child Neurology. 2014;9(4):40-48. doi:10.17650/20738803-2014-9-4-40-48. [Article in Russian].

4. Guidelines for the diagnosis and management of syncope (version 2009). Task Force for the Diagnosis and Management of Syncope; European Society of Cardiology (ESC); European Heart Rhythm Association (EHRA); Heart Failure Association (HFA); Heart Rhythm Society (HRS); Moya A, Sutton R, Ammirati F, Blanc JJ, Brignole M, Dahm JB, et al. Eur Heart J. 2009;30(21):2631-71. doi: 10.1093/eurheartj/ehp298

5. Mathias CJ, Mallipeddi R, Bleasdale-Barr K. Symptoms associated with orthostatic hypotension in pure autonomic failure and multiple system atrophy. J Neurol. 1999;246(10):893-8.

6. Sheldon R1, Rose S, Ritchie D, Connolly SJ, Koshman ML, Lee MA, et al. Historical criteria that distinguish syncope from seizures. J Am Coll Cardiol. 2002;40(1):142-8.

7. Duplyakov D, Gavrilova E, Golovina G, Lyukshina N, Sysuenkova E, Shagrova E, et al. Prevalence of epileptiform findings on routine EEG and its influence on the result of head-up tilt test in patients with neurocardiogenic syncope. Eur Heart J. 2007;28 (Suppl):640.

8. Hoefnagels WA, Padberg GW, Overweg J, van der Velde EA, Roos RA. Transient loss of consciousness: the value of the history for distinguishing seizure from syncope. J Neurol. 1991;238(1):39-43.

9. Shnayder NA, Sadikova AV, Nikulina SYu, Shnayder VA. [Sudden unexpected death in epilepsy]. Medicine of Extreme Situations. 2011;36(2):54-64. [Article in Russian].

10. van Dijk JG, Sheldon $\mathrm{R}$. Is there any point to vasovagal syncope? Clin Auton Res. 2008;18:167-9. doi: 10.1007/s10286-008-0484-x. 